\section{Founder effect}

\section{J. Arnemann}

Abteilung Molekulargenetik, Labor Dr. Wisplinghoff, Köln, Deutschland

\section{Synonym(e) Gründereffekt}

\section{Englischer Begriff founder effect}

Definition Der Gründereffekt oder „founder effect“ beschreibt die Manifestation einer genetischen Variation oder auch Neumutation aufgrund einer begrenzten Anzahl an Allelen in einer Gründerpopulation.

Beschreibung Gründerpopulationen fand man in Deutschland und Europa in relativ unzugänglichen Bergregionen oder nur dünn besiedelten Landstrichen, wo die Bevölkerung einen geringen Austausch mit anderen hatte und bei den Ehen oftmals ein hoher Anteil an Verwandtschaften vorlag. Dies war die Grundlage, dass sich meist rezessive, seltener auch dominante Mutationen im Genpool festigen konnten und teilweise neue Krankheitsbilder oder Fehlbildungen entstehen ließ, die in der übrigen Bevölkerung zunächst unbekannt waren. Beispiele für einen Gründereffekt findet sich in der rezessiven adulten Laktoseintoleranz in der ursprünglich nordeuropäischen, Milchwirtschaft betreibenden Bevölkerung oder die starke Vermehrung der dominanten ChoreaHuntington-Erkrankung am Maracaibo-See in Venezuela, ausgehend von einer Frau mit 10 Kindern vor über 200 Jahren.

\section{Literatur}

Claramunt $\mathrm{R}$ et al (2005) Genetics of Charcot-Marie-Tooth disease type 4A: mutations, inheritance, phenotypic variability, and founder effect. J Med Genet 242:358-365 